\title{
An Evaluation of Two Advanced Turbulence Models for Simulating the Flow and Dispersion Around Buildings
}

S.T. Chan and D.E. Stevens

This article was submitted to

Millennium NATO/CCMS International Technical Meeting on Air Pollution Modeling and Its Application Boulder, CO

May 15-19, 2000

Lawrence

Livermore

National

Laboratory

March 14, 2000 


\section{DISCLAIMER}

This document was prepared as an account of work sponsored by an agency of the United States Government. Neither the United States Government nor the University of California nor any of their employees, makes any warranty, express or implied, or assumes any legal liability or responsibility for the accuracy, completeness, or usefulness of any information, apparatus, product, or process disclosed, or represents that its use would not infringe privately owned rights. Reference herein to any specific commercial product, process, or service by trade name, trademark, manufacturer, or otherwise, does not necessarily constitute or imply its endorsement, recommendation, or favoring by the United States Government or the University of California. The views and opinions of authors expressed herein do not necessarily state or reflect those of the United States Government or the University of California, and shall not be used for advertising or product endorsement purposes.

This is a preprint of a paper intended for publication in a journal or proceedings. Since changes may be made before publication, this preprint is made available with the understanding that it will not be cited or reproduced without the permission of the author.

This report has been reproduced directly from the best available copy.

Available to DOE and DOE contractors from the

Office of Scientific and Technical Information

P.O. Box 62, Oak Ridge, TN 37831

Prices available from (423) 576-8401

http:/ / apollo.osti.gov/bridge/

Available to the public from the National Technical Information Service

U.S. Department of Commerce 5285 Port Royal Rd., Springfield, VA 22161 http://www.ntis.gov/

OR

Lawrence Livermore National Laboratory Technical Information Department's Digital Library http://www.llnl.gov/tid/Library.html 


\title{
AN EVALUATION OF TWO ADVANCED TURBULENCE MODELS FOR SIMULATING THE FLOW AND DISPERSION AROUND BUILDINGS
}

\author{
S.T. Chan and D.E. Stevens \\ Lawrence Livermore National Laboratory \\ Livermore, CA 94551
}

\section{INTRODUCTION}

Numerical modeling of airflow and pollutant dispersion around buildings is a challenging task due to the geometrical variations of buildings and the extremely complex flow created by such surface-mounted obstacles. The airflow around buildings inevitably involves impingement and separation regions, building wakes with multiple vortices, and jetting effects in street canyons. The interference from adjacent buildings further complicates the flow and dispersion patterns. Thus accurate simulations of building-scale transport phenomena requires not only appropriate physics submodels but also significant computing resources. We have developed an efficient, high resolution CFD model for simulating chemical and biological releases around buildings. Our primary goal is to support incident response and preparedness in emergency response planning and vulnerability analysis.

Our model, FEM3MP, solves the three-dimensional, time-dependent Navier-Stokes equations. The numerical algorithm uses an innovative finite element approach to accurately represent complex building shapes and a fully implicit projection method for efficient time integration (Gresho and Chan, 1998). For turbulence parameterization, we have implemented, in addition to a $\mathrm{k}-\varepsilon$ turbulence submodel, a nonlinear eddy viscosity (NEV) and a large eddy simulation (LES) turbulence submodels. Other model physics, including UV radiation decay, aerosols, surface energy budget, and tree canopy effects, have also been implemented. Our model has been developed to run on both the serial and massively parallel computer platforms.

Model validation is a fundamental component of establishing the credibility of this model for use in the assessment of flow and dispersion of hazardous agents around buildings. We have performed model validations using, among others, the tow-tank experimental data obtained from flow and dispersion past a cubical building (Zhang et al., 1996) and also similar data around a 2-D array of buildings in a wind tunnel (Brown et al., 2000).

In this paper, we will describe briefly the salient features of our model, present and discuss results from a model-data comparison study. Due to space limitations, only results for the cubical building, with a focus on the performance of the NEV and LES turbulence submodels, are presented in this paper. Additional results will be presented at the conference.

\section{NUMERICAL MODEL}

\section{Governing Equations}

The equations modeled in this validation study are a subset of the generalized anelastic equations available to FEM3MP. These equations, written in Cartesian tensor form, are: 


$$
\begin{aligned}
& \frac{\partial}{\partial t} u_{i}+u_{j} \frac{\partial u_{i}}{\partial x_{j}}=-\frac{\partial p}{\partial x_{i}}+v \frac{\partial^{2} u_{i}}{\partial x_{j} \partial x_{j}}+\frac{\partial}{\partial x_{j}}\left(-\overline{u_{i}^{\prime} u_{j}^{\prime}}\right) \\
& \frac{\partial}{\partial x_{j}} u_{j}=0, \\
& \frac{\partial c}{\partial t}+u_{j} \frac{\partial c}{\partial x_{j}}=v \frac{\partial^{2} c}{\partial x_{j} \partial x_{j}}+\frac{\partial}{\partial x_{j}}\left(-\overline{u_{j}^{\prime} c^{\prime}}\right)
\end{aligned}
$$

In the above equations, $u_{i}$ is the $i$-th component of the mean velocity, $c$ is the mean tracer concentration, $p$ is the dynamic pressure, $v$ is the kinematic viscosity, $u_{i}^{\prime} u_{j}^{\prime}$ are turbulent stresses, and $\overline{u_{j}^{\prime} c^{\prime}}$ is the mass flux of the tracer. The RANS (Reynolds-Averaged Navier-Stokes) and LES approaches are used to parameterize the turbulent stresses and mass fluxes.

The above set of governing equations are firstly discretized in space by the Galerkin finite element method, with piecewise constant representation for pressure and trilinear approximations for other field variables, to obtain a couple system of nonlinear first-order ordinary differential equations. In order to solve cost-effectively large three-dimensional problems, we employ the fully implicit projection method developed by Gresho and Chan (1998). With the method, the coupled system of equations are segregated and solved sequentially for each of the field variables via efficient iterative solvers.

\section{RANS Turbulence Submodel}

In our RANS approach, we used a nonlinear eddy viscosity (NEV) turbulence submodel developed by Suga (1995). This 3-equation turbulence submodel has many desirable properties, including anisotropy, a cubic constitutive law, and no need for wall functions. Since the model has been derived from the Reynolds stress closure models, it retains many of their attributes - but at a significantly reduced computational cost.

The crux of the submodel involves the following three fairly complex, coupled equations, with details available in Suga (1995), Gresho and Chan (1998):

$$
\begin{aligned}
& \frac{\partial k}{\partial t}+u_{j} \frac{\partial k}{\partial x_{j}}=\frac{\partial}{\partial x_{i}}\left[\left(v \delta_{i j}+0.22 f_{g} \overline{u_{i}^{\prime} u_{j}^{\prime}} \frac{k}{\bar{\varepsilon}}\right) \frac{\partial k}{\partial x_{j}}\right]+P_{K}-\varepsilon, \\
& \frac{\partial \bar{\varepsilon}}{\partial t}+u_{j} \frac{\partial \bar{\varepsilon}}{\partial x_{j}}=\frac{\partial}{\partial x_{i}}\left[\left(v \delta_{i j}+0.18 f_{g} \overline{u_{i}^{\prime} u_{j}^{\prime}} \frac{k}{\bar{\varepsilon}}\right) \frac{\partial \bar{\varepsilon}}{\partial x_{j}}\right]+\left(1.2 P_{K}-C_{\varepsilon} \bar{\varepsilon}\right) \frac{\bar{\varepsilon}}{k}+P_{\varepsilon}, \\
& \frac{\partial A_{2}}{\partial t}+u_{j} \frac{\partial A_{2}}{\partial x_{j}}=\frac{\partial}{\partial x_{i}}\left[\left(v \delta_{i j}+0.22 f_{g} \overline{u_{i}^{\prime} u_{j}^{\prime}} \frac{k}{\bar{\varepsilon}}\right) \frac{\partial A_{2}}{\partial x_{j}}\right]+\frac{a_{i j}}{k}\left[P_{i j}-\varepsilon_{i j}+\phi_{i j}\right]-\frac{2 A_{2}}{k} P_{K} \text {. }
\end{aligned}
$$

In the above equations, $k$ is the turbulent kinetic energy (TKE), $\bar{\varepsilon}$ is the isotropic dissipation rate, and $A_{2}$ is the second invariant of the dimensionless anisotropic Reynolds stress tensor.

The Reynolds stresses are defined by a cubic constitutive relationship

$$
\begin{aligned}
\overline{u_{i}^{\prime} u_{j}^{\prime}} & =\frac{2}{3} k \delta_{i j}-v_{T} S_{i j}+\frac{v_{T} k}{\bar{\varepsilon}}\left\{c_{1}\left(S_{i k} S_{k j}-\frac{1}{3} S_{k \ell} S_{k \ell} \delta_{i j}\right)\right. \\
& +c_{2}\left(\Omega_{i k} S_{k j}+\Omega_{j k} S_{k i}\right)+c_{3}\left(\Omega_{i k} \Omega_{j k}-\frac{1}{3} \Omega_{\ell k} \Omega_{\ell k} \delta_{i j}\right) \\
& \left.+\frac{k}{\bar{\varepsilon}}\left[c_{4}\left(S_{k i} \Omega_{\ell j}+S_{k j} \Omega_{\ell i}\right) S_{k \ell}+c_{5} S_{i j} S_{k \ell} S_{k \ell}\right]+c_{6} S_{i j} \Omega_{k \ell} \Omega_{k \ell}\right\},
\end{aligned}
$$

in which $S_{i j}$ is the mean strain rate tensor and $\Omega_{i j}$ is the mean rotation tensor. The isotropic turbulent eddy viscosity is defined as 


$$
v_{T}=c_{\mu} f_{\mu} k^{2} / \bar{\varepsilon}
$$

where $c_{\mu}$ is a turbulent viscosity parameter and $f_{\mu}$ is a wall damping function, both being functions of the turbulence variables $\mathrm{k}, \bar{\varepsilon}$, and $\mathrm{A}_{2}$.

\section{LES Subgrid-Scale Modeling}

The role of the turbulence submodel in an LES simulation is to parameterize the effect of motions that cannot be resolved by the computational mesh. The one used here was developed by Smagorinsky (1963), with the subgrid TKE, e, and the eddy viscosity given by,

$$
e^{1 / 2}=\frac{c_{s}^{2}}{c_{m}} l\left|S_{i j}\right|, \quad K_{m}=c_{s}^{2} l^{2}\left|S_{i j}\right| .
$$

Here $c_{s}$ and $c_{m}$ are empirical constants with values of 0.1 and 0.3 in this study. The subgrid scale fluxes are related to the gradients of the mean variables as,

$$
\overline{u_{i} u_{j}^{\prime}}=-K_{m} S_{i j}, \quad \overline{u_{j} c^{\prime}}=-K_{m} \frac{\partial c}{\partial x_{j}},
$$

Although simpler conceptually, the properties of the flow are more difficult to diagnose for an LES than a RANS simulation. For example, TKE is computed explicitly in a RANS model. For an LES calculation, one must first generate a mean velocity field and the deviations from this field, square and average again to generate the resolved TKE. Averaging is also needed for the sub-grid TKE, since the sum of these two quantities has to be used for comparison with RANS and the experimental results.

One problem associated with LES turbulence models is their inability to model near wall behavior. For this study, we used free-slip on the top and bottom boundary to maximize turbulent dispersion. Also, $c_{S}=0.1$, instead of 0.23 (a value typically used in atmospheric LES), was used to enhance crosswind dispersion.

\section{MODEL-DATA COMPARISON}

This paper presents a validation study of flow and dispersion around a cubical building, using both the RANS and LES approaches. There have been many laboratory experiments and numerical studies performed on this flow. The fidelity of our model with respect to the flow field is judged by comparison with the laboratory measurements of Martinuzzi and Tropea (1993) and the LES results of Shah (1998). For dispersion assessment, we use the results from a study by Zhang et al. (1996) for a continuous, ground-level tracer release behind the cube.

The computational domain used in our numerical simulations is $10 \mathrm{H}$ by $7 \mathrm{H}$ by $2 \mathrm{H}, \mathrm{H}$ being the cube height, in the longitudinal, lateral, and vertical directions, respectively. The origin is situated at the center of the cube, with the inflow at $\mathrm{x}=-3.5 \mathrm{H}$.

Since the RANS assumes a steady state solution and the geometry is symmetric, only one-half of the domain was simulated. This enables the RANS to use a small mesh of $96 \times 37 \times 30$ nodal points. No slip boundary condition was used along the channel walls and on surfaces of the cube. The mean velocity of a fully-developed channel flow of average velocity of $0.6 \mathrm{~m} / \mathrm{s}$ was specified on the inlet plane. The entire simulation took about 25 hours on 2-3 processors of a shared-memory multi-processor DEC ALPHA to integrate 1200 time steps.

The LES simulation used a computational mesh of $129 \times 65 \times 65$ nodal points and integrated for 1920 time steps. This generated a time series of data for 57.6 nondimensional time units, $t^{*}=U_{o}$ $\mathrm{t} / \mathrm{H}$. A uniform inflow velocity $\mathrm{U}_{\mathrm{o}}=0.6 \mathrm{~m} / \mathrm{s}$ was used to match the inflow characteristics of Zhang et al. (1996). The LES calculation requires roughly an order of magnitude more effort than the RANS simulation. However, due to the use of parallel computing using 64 IBM processors, this simulation took only 18 hours to complete.

The LES simulation was integrated for 40 non-dimensional time units before averaging starts. At this point, the mean concentration in the domain has stabilized and the flow has settled down in alternately shedding pairs of vortices behind the cube. Our simulation generated a pair of vortices 
every 7.5 non-dimensional time units, which compares reasonably well with an estimated value of 8.5 by Shah's LES model.

In Fig. 1, time-averaged streamlines are compared. The main features of all flow fields are similar. They include the separation zones in front of the cube, on the roof and the two sides (not shown), a primary recirculation zone in the wake, and a pair of counter-rotating vortices on the horizontal plane (not shown). The predicted reattachment length is 1.85 by the RANS model and is 1.55 by the LES model. These values agree well with the value of 1.68 measured by Martinuzzi and Tropea (1993), and with the value of 1.64 predicted by Shah (1998). The present model results compare much better than the value of 2.85 predicted by the simpler k- $\varepsilon$ turbulence model (Chan and Lee, 1999) and the values between 2.68 and 3.4 predicted by variants of two-layer $k-\varepsilon$ models (Lakehal and Rodi, 1997).
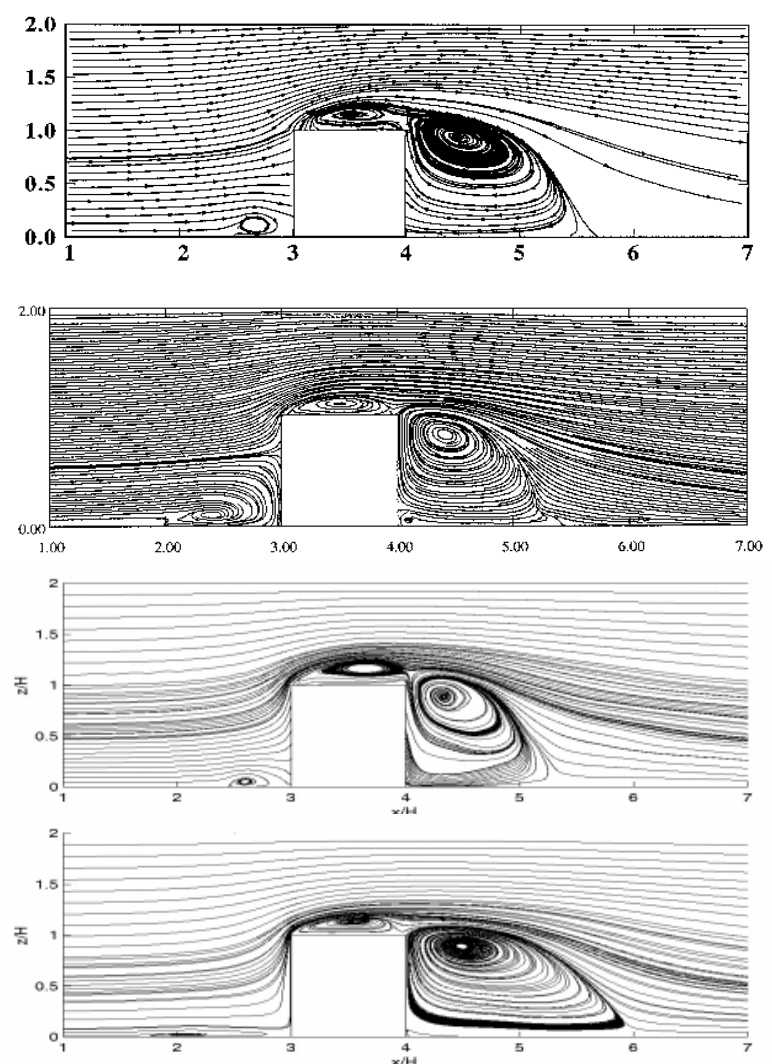

Figure 1

Figure 1: Comparison of time-averaged streamlines along the symmetry plane for the experimental results of martinuzzi and Tropea (top), Shah (top middle), FEM3MP LES (bottom middle), and RANS (bottom) 

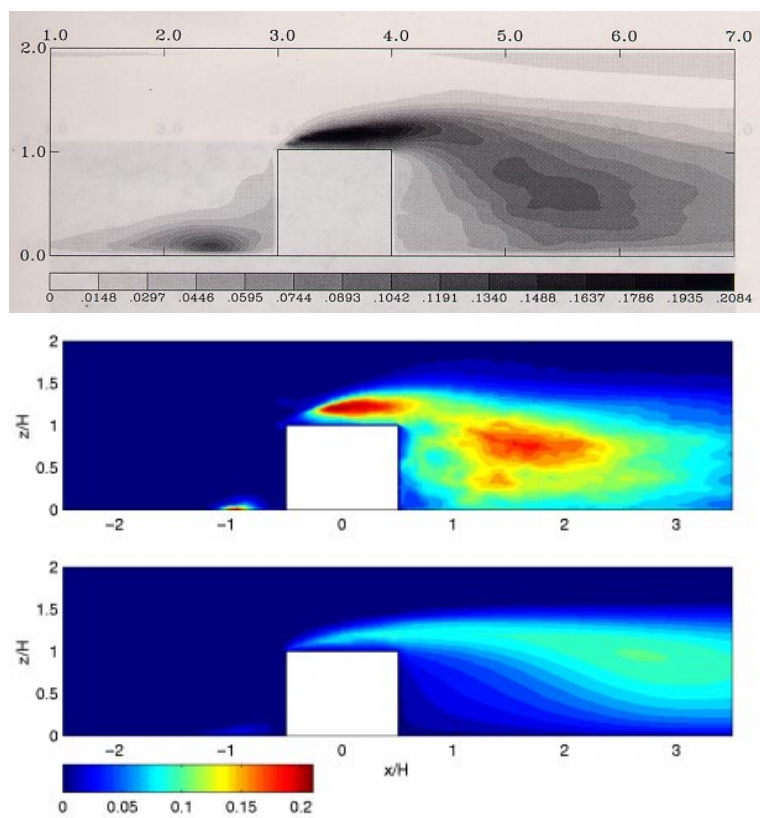

Figure 2

Figure 2: Comparison of normalized TKE along the symmetry plane for Shah's LES (top), FEM3MP LES (middle), and RANS (bottom).

In Fig. 2, the predicted TKE fields on the vertical plane of symmetry are compared with that obtained by Shah (1998), who used an LES model, a finer grid of 192x64x96, and a fully developed channel flow as inlet boundary condition. The TKE fields from both LES models tend to be larger and extend closer to the roof than that from the RANS. Our LES tends to predict higher TKE behind the cube, while Shah's has a bigger region of large TKE values in front of the cube. Besides the difference in inlet velocity profiles, Shah used no slip boundary conditions, while we used free-slip boundary conditions.

In Fig. 3, we compare the predicted turbulent kinetic energy profiles at selected locations with the data of Martinuzzi and Tropea (1993). These profiles indicate that both turbulence submodels are able to predict the bulk flow features observed in the experiment. Overall, the LES predicts the TKE better than the RANS. The small discrepancies near the top and bottom of the domain from the LES model are generated by the free-slip boundary conditions. Both models predict peak TKE values at approximately the correct locations.

The results of Zhang et al. (1996) are used herein to assess the accuracy of concentration patterns predicted by our model. These results were created with a tow-tank in which a cube of $0.1 \mathrm{~m}$ high mounted to a plate was moved at $0.6 \mathrm{~m} / \mathrm{s}$ through a water tank of $18 \mathrm{~m}$ long and $1 \mathrm{~m} \times 1 \mathrm{~m}$ in cross section. The dispersion was generated by a ground-level, continuous source placed at $0.25 \mathrm{H}$ behind the cube. The experiment showed that the tracer is entrained into the recirculating eddy behind the cube and dispersed downstream. The plume quickly becomes much wider than the cube width within a few block heights downstream. 

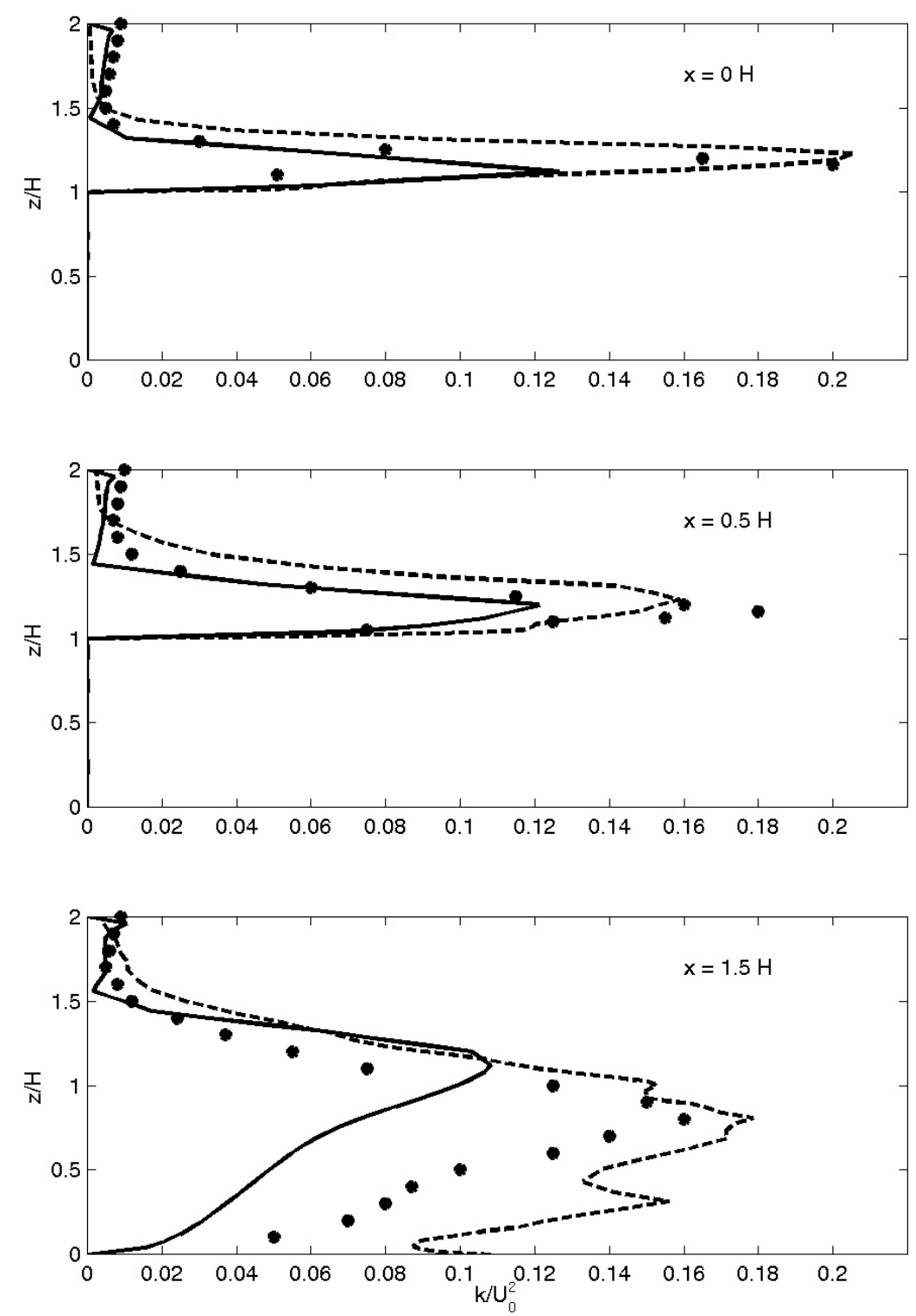

Figure 3

Figure 3: Comparison of normalized TKE for experiment (dots), LES (dashed line), and RANS (solid line) at three vertical profiles along the symmetry plane.

In Figs. 4 and 5, concentration patterns on the floor and the plane of symmetry are displayed. The contours are normalized concentration $\chi=\mathrm{CU}_{\mathrm{o}}{ }^{2} \mathrm{H} / \mathrm{Q}$, where $\mathrm{C}$ is concentration and $\mathrm{Q}$ is the source flux. An interesting feature of these plots is that the instantaneous plume of LES looks very similar to the RANS plume in terms of width and height, except for a small horizontal translation downstream. The higher concentration contours are very similar among the three panels, indicating that both the RANS and LES simulations are performing well for the bulk of the dispersion. However, the RANS simulation is missing the wavy structure of the dispersion patterns shown in Fig. 4 and the eddy mixing at the top edge of the plume shown in Fig. 5. In addition, the LES plume has a wider spread in mean concentration, which was also observed in the experiment as a result of vortex shedding generated by the cube. 

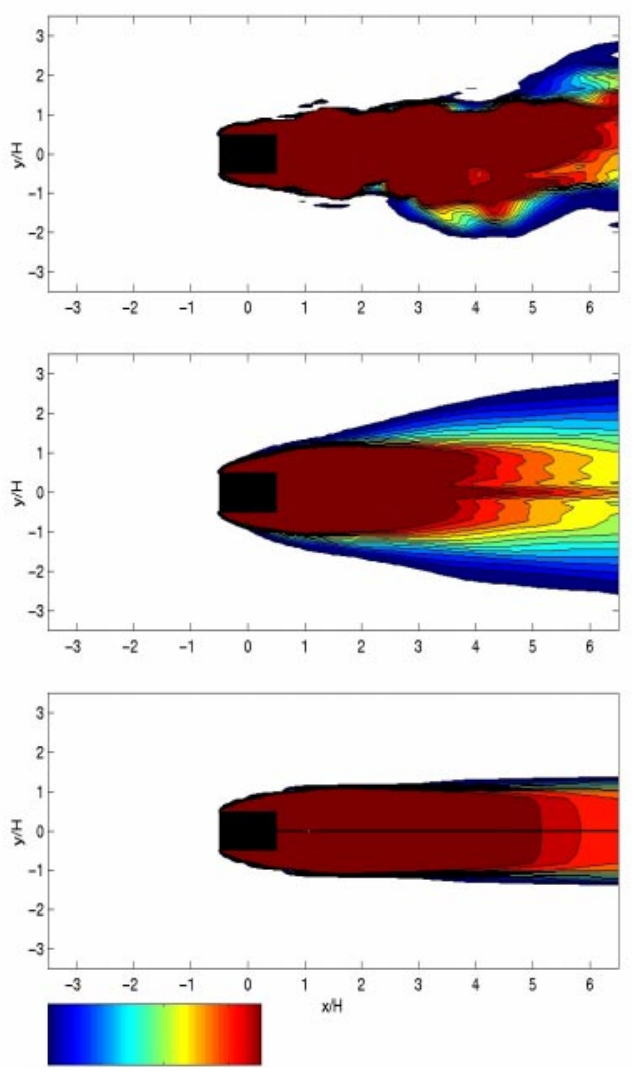

Figure 4

Figure 4: Tracer concentrations along the floor plane showing the instantaneous pattern form the LES (top), the mean from the LES simulation (middle), and from the RANS simulation (bottom).
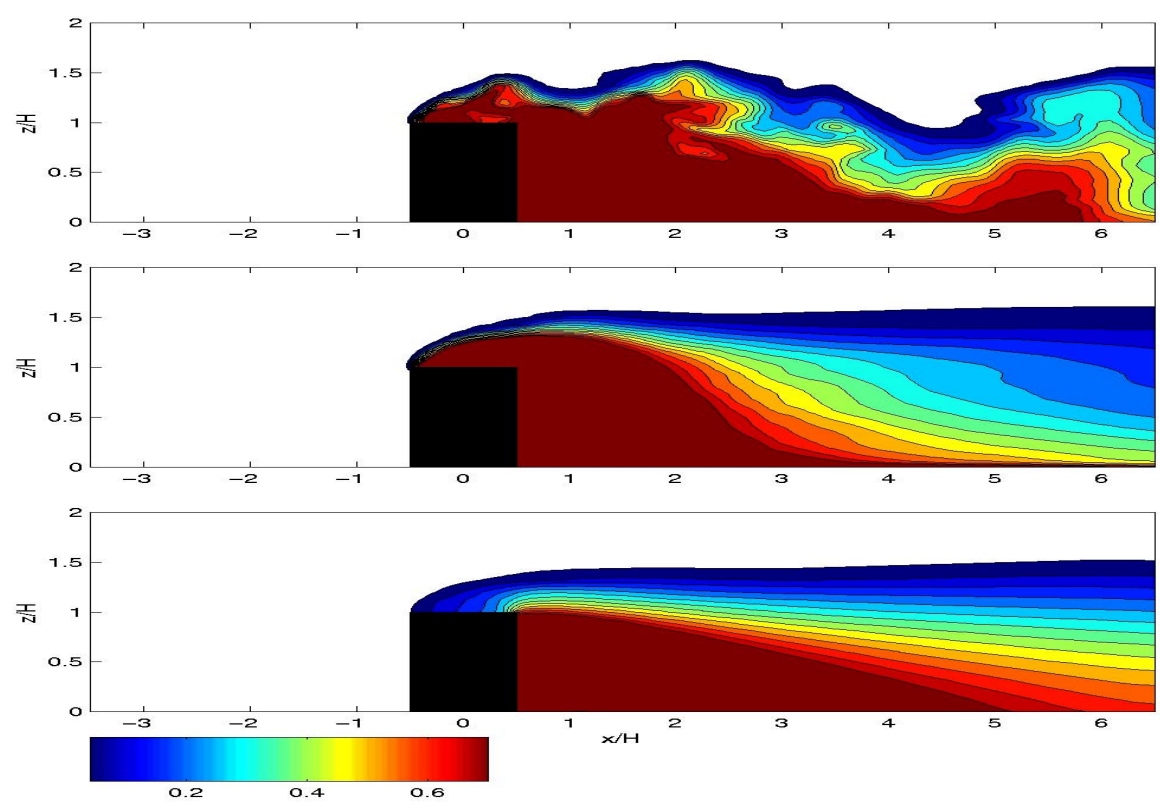

Figure 5

Figure 5: Tracer concentrations along the symmetry plane showing the instantaneous pattern from the LES (top), the mean from the LES simulation (middle), and from the RANS simulation (bottom). 

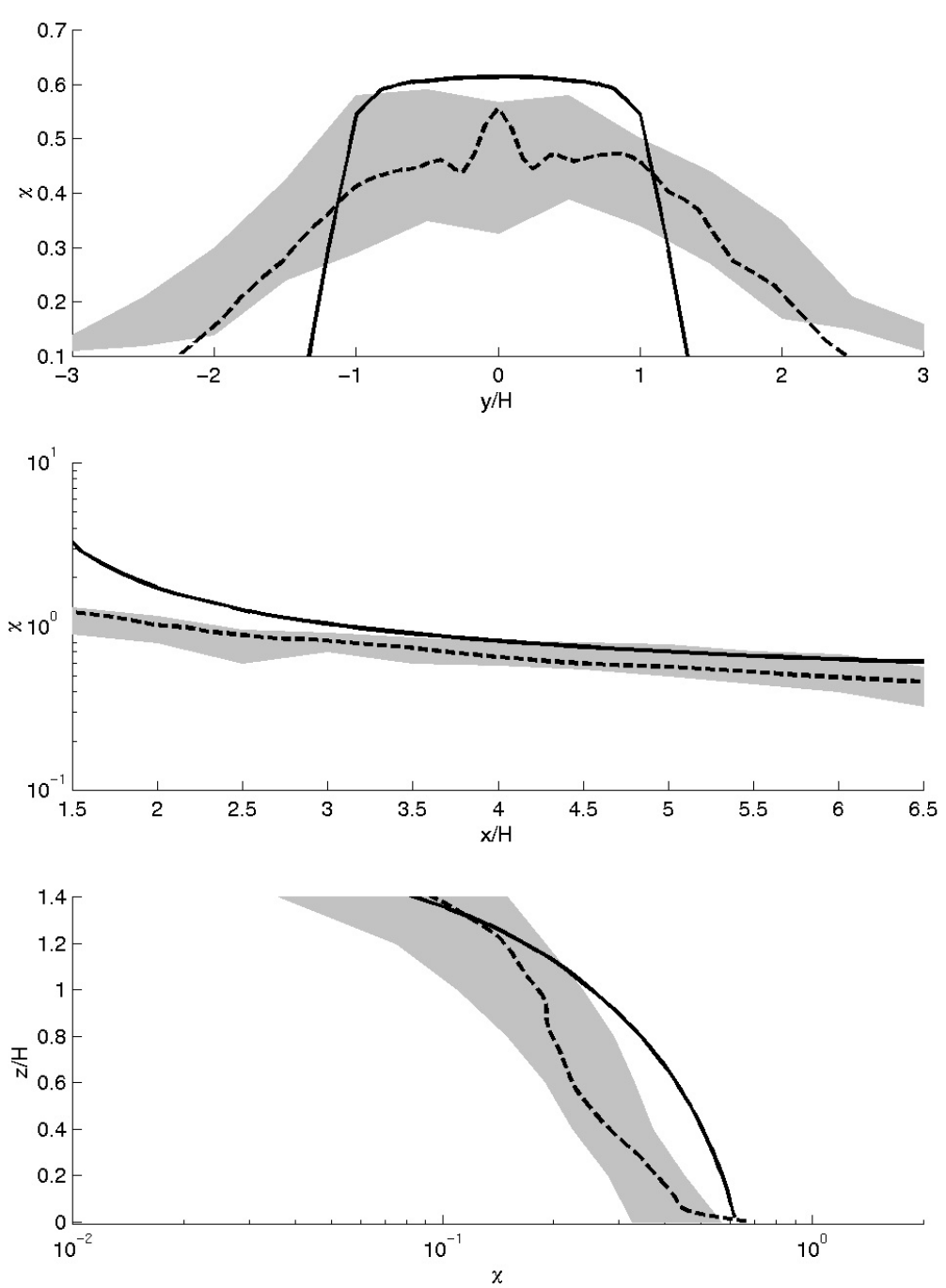

Figure 6

In Fig. 6, we compare the predicted concentration profiles with measurements (shaded area) along three selected lines. The top plot shows the LES capturing the horizontal dispersion at the outflow as observed in the experiment. The middle plot shows the LES and RANS agreeing with experiments along the floor of the symmetry plane. Both of these plots support our observation that the RANS plume is similar to a realization of the LES plume. The bottom plot shows the vertical concentration profile centered in the middle of the outflow plane. This plot further supports this idea, since both RANS and mean values of LES have similar vertical extent as they agree towards the top of the profile. However, the lack of vortex shedding in the RANS simulation has resulted in higher concentrations near the bottom of the profile.

Figure 6: Comparison of the mean concentrations along three lines: the horizontal line at outflow (top), the horizontal line at the bottom of the symmetry plane (middle), and the vertical line at outflow (bottom). LES (dashed line), RANS (solid line). 


\section{SUMMARY}

We have presented a validation for our model through simulating the flow and dispersion around a cubical building. It was found that the LES model yields more accurate results but with roughly an order of magnitude increase in computational cost. Several conclusions can be drawn from this study:

1. Instantaneous snapshots of concentration from the LES model have similar vertical depth and width as the RANS. This indicates RANS is producing a reasonable plume, but without the spatial fluctuation associated with vortex shedding in the experimentally observed wake.

2. The RANS and LES yield similar mean velocity fields near the building. If mean velocity fields are sufficient and decoupled from the advected species, the RANS solution is the more economical method.

3. The LES can capture turbulent variations that the RANS does not model. For situations in which the source lasts sufficiently long, LES simulates more accurately the width of the dispersion than RANS does. This is useful for situations where accurate species concentrations in both space and time are important.

\section{ACKNOWLEDGMENTS}

This work was performed under the auspices of the U.S. Department of Energy by University of California Lawrence Livermore National Laboratory under contract number W-7405-ENG-48.

\section{REFERENCES}

Brown, M.J., R.E. Lawson, D.S. DeCrox, and R.L. Lee, 2000, Mean flow and turbulence measurements around a 2-D array of buildings in a wind tunnel, 11th J. AMS/AWMA Conf. on the Appl. of Air Poll. Met., Long Beach, CA., 35-40.

Chan, S.T.and R.L. Lee, 1999, A model for simulating airflow and pollutant dispersion around buildings: Air Pollution VII, WIT Press, 39-48.

Gresho, P.M. and S.T. Chan, 1998, Projection 2 goes turbulent - and fully implicit, Int. J. of Comp. Fluid Dyn. 9:249-272.

Lakehal D. and W. Rodi, 1997, Calculation of the flow past a surface-mounted cube with two-layer turbulence models, J. Wind Eng. Ind. Aerodyn. 67 \& 68:65-78.

Martinuzzi, R. and C. Tropea, 1993, The flow around surface-mounted, prismatic obstacles placed in a fully developed channel flow, J Fluids Eng. 115:85-92.

Shah, K.B., 1998, Large eddy simulations of flow past a cubic obstacle. Ph.D. thesis, Stanford University.

Smagorinsky, J., 1963, General circulation experiments with the primitive equations: 1. The basic experiment, Mon. Wea. Rev. 121:1847-1859.

Suga, K., 1995, Development and application of a nonlinear eddy viscosity model sensitized to stress and strain invariants. Ph.D. thesis, UMIST, Manchester, UK.

Zhang, Y.S., S. Arya, and W. Snyder, 1996, A comparison of numerical and physical modeling of stable atmospheric flow and dispersion around a cubical building, Atm. Env. 30:1327-1345. 